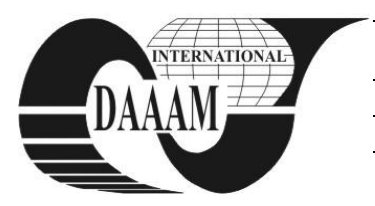

Annals of DAAAM for 2012 \& Proceedings of the 23rd International DAAAM Symposium, Volume 23, No.1, ISSN 2304-1382 ISBN 978-3-901509-91-9, CDROM version, Ed. B. Katalinic, Published by DAAAM International, Vienna, Austria, EU, 2012 Make Harmony between Technology and Nature, and Your Mind will Fly Free as a Bird

\title{
POSSIBILITY OF IMPLEMENTATION OF THE ADVANCED FIRE DETECTION INFORMATION SYSTEM FOR THE FRUSHKA GORA NATIONAL PARK
}

FROST, P. E.; SAKULSKI, D[usan]; COSIC, D[jordje]; POPOV, S[rdjan] \& NOVAKOVIC, T[anja]

\begin{abstract}
Every summer season an open space fires takes lives and result in huge economic, environmental and cultural losses. Timing is one of the most critical parameters. To spot fire as soon as possible, to locate fire as accurate as possible, to react as quick and efficient as possible, are some of the imperatives to save lives and minimise damage. This paper presents an ongoing development of the Advanced Fire Location and Information System. It was developed for South Africa and is in the process of improvement and modification to accommodate user requirements of "Fruška Gora" National Park, with the intention to expand it to all countries in southern and southeastern Europe. Fire information systems utilises MODIS and MSG satellite images to detect and locate fire, and e-mail and SMS messaging to deliver critical information to disaster risk managers and decision makers. It is result of the cooperation between CSIR MERAKA Institute (South Africa) and Faculty of Technical Sciences, University of Novi Sad (Serbia).
\end{abstract}

Keywords: Fire, fire detection, fire information, MODIS, MSG, Remote sensing, "Fruška Gora" National Park.

\section{INTRODUCTION}

Open space fire prevention, mitigation and early warning system, as an integral part of the disaster risk reduction, do not exists in Serbia. Those are very reasons why impact and damages regarding open space fires are high. Especially during very hot and dry spring and summer seasons, like a recent season 2011/2012, when vegetation is dry, and very often wind is strong, costly damage is inevitable:

- "One billion Euro burned out:" (Source: TANJUG, 08.12.2011)

- "Nine fires during two days at the Fruška Gora National Park" (Source: TANJUG, 17.03.2012)

- "760 fires during three days period: There were 760 fire incidents for the past three days over Serbian territory, resulting in three lives lost." (Source: BETA, 19.03.2012).

In situation like these, time is of the crucial importance. Reaction speed and coordination are the most significant factors. The most common denominator for those is availability of information related to fire, such as:

- Timing and positioning of fire started

- Temperature, pressure and wind information
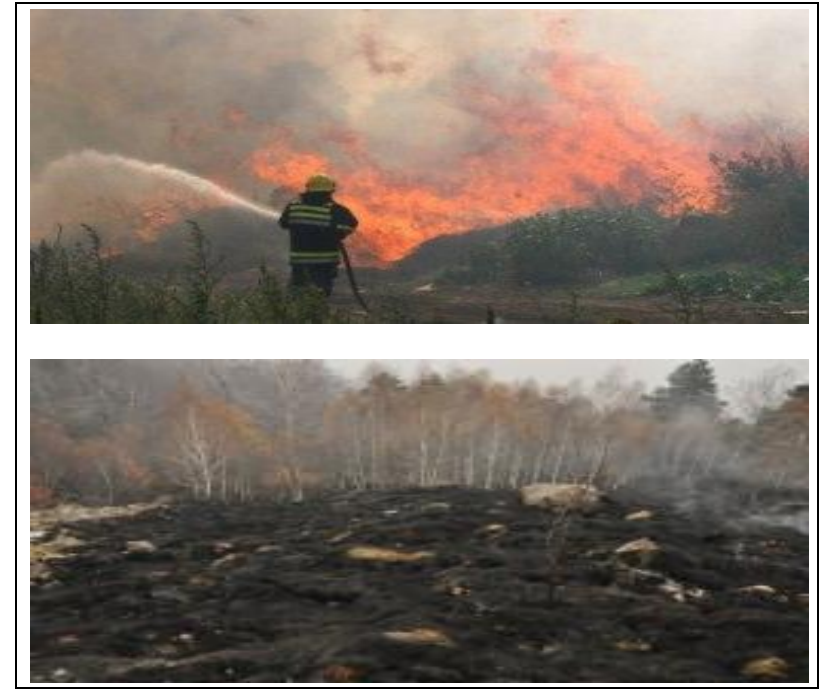

Fig. 1. Recent fires across Serbia (Source: BETA, TANJUG)

- Vegetation cover

- Vegetation dryness (fire load)

- Water logs positioning

- Proximity of the residential and/or industrial facilities.

\section{IMPORTANCE OF FIRE DETECTION AND ALERT}

For many African countries, like South Africa,fires in South Africa is part of the landscape and most plants are adapted to fire, if not dependent on it for germination of their seeds. The first recording of a veld fire was

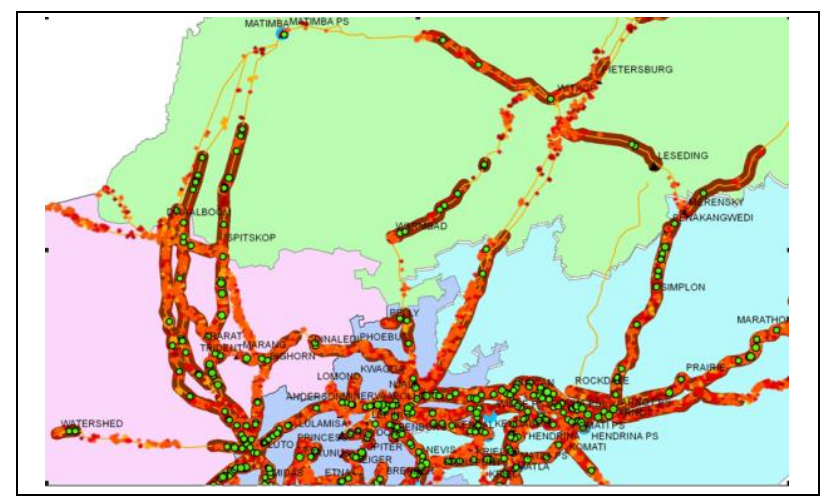

Fig. 2. The historic data of the occurrence of fire (in red) and fire faults (in green) indicate the high risk areas for each line (shown in brown), for the central part of South Africa. (Source: ESKOM) 
probably done by Bartolomeu Dias when he became the first European to round the Cape in 1488. Not everyone has benefit of an open space (veld) fires occurring every Year. ESKOM, national electricity generator and supplier, count huge damage caused by veld fires under the transmission lines.

Although transient faults on the transmission system are of extremely short duration measured in milliseconds, their effect and cost to the utility and its customers can be huge. According to a study carried out in 2001 by EPRI, poor power quality costs the American economy between $\$ 15$ and $\$ 25$ thousand million per annum [4]. This Fig. is likely to rise as the sophistication of the customer increases in the future. In South Africa studies show that the cost is highly variable and depends on the location of the customer relative to the fault. Cost estimates of damage experienced by customers range between ZAR5000 and ZAR150000 per dip [6].

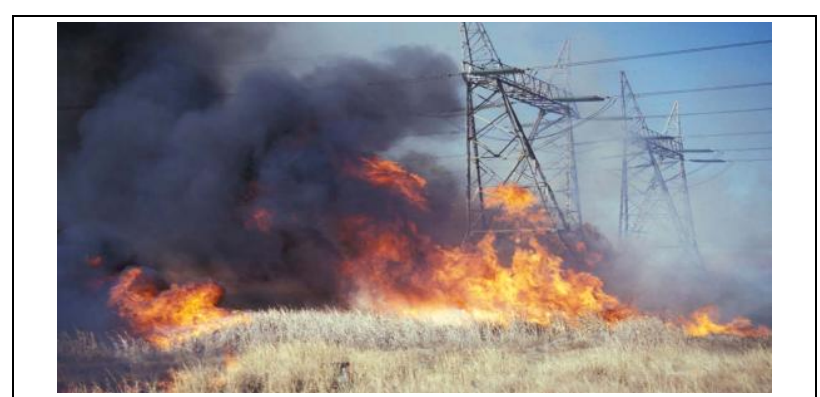

Fig. 3. Grass fires burning under the ESKOM power lines (Source: ESKOM)

Fires under transmission lines constitute about $20 \%$ of the total. It must be pointed out that the fires referred to here are not the high intensity fires of Australia or California recently reported in the media, but rather low intensity grass fires that produce enough ionized air to break down the isolation of the air. It also needs to be pointed out that the ionized air and not the smoke particles in the air, is responsible for the breakdown in isolation of the air [5].

The launching of the Aqua and Terra satellites with the Moderate Resolution Imaging Spectroradiometer (MODIS) by NASA in 1999 and 2002 provided the world with a tool to be used inter alia in fire tracking. The University of Maryland (USA) demonstrated the successful use of this data in the mapping of fires across the globe.

Detection of fires are influence by the following factors; satellite overpass time (MODIS), scan angle, biome, sun position, land surface temperature, cloud cover, etc.

-MODIS fires - Fires as small as $50 \mathrm{~m}^{2}$ can be detected under optimal conditions, but average detection size is around $100 \mathrm{~m}^{2}$.

-MSG fires - MSG is a coarse resolution satellite $36000 \mathrm{~km}$ from earth. Validation in the USA on similar satellites has shown detectable sizes in the region of 500 $\mathrm{m}^{2}$ again taking the influential factors into account.
In order to detect fires in near real-time, it requires that the data is downloaded as the satellite passes over, processed and broadcast to the user [2].This was not possible with the Maryland system. When the South African Department of Agriculture purchased the satellite data and a MODIS antenna at Hartbeeshoek (near Pretoria) in 2003, this fast access to the satellite became a reality to ESKOM.

In early 2004 ESKOM and the Satellite Application Centre (SAC) of the Council of Scientific and Industrial Research (CSIR), launched a research project to demonstrate the ability to track active fires by using polar orbiting satellites. MODIS being medium-resolution scanner enables four updates daily with a $1 \mathrm{~km} 2$ resolution. The detection of grass fires as small as $0.25 \mathrm{ha}$ is possible with this data [7]

ESKOM required information on fires every 15 minutes and whilst the MODIS data was sufficiently high in spatial resolution, the temporal resolution was less than satisfactory. Consequently the CSIR proposed the use of the Spinning Enhanced Visible and Infrared Imager (SEVIRI) sensor on board the Meteosat Second Generation (MSG) satellite. This satellite is in geostationary orbit above the equator and the SEVIRI sensor transmits data every 15 minutes. MSG data is observed at a spatial resolution of $5 \mathrm{~km} 2$ [1]

Once the processing of the hot spots have been completed the information is published on a website, or e-mail alerts are sent. Due to the fact that the ESKOM's field personnel are normally out on patrols, the idea emerged to send text message warning of fires to their mobile phones.

\section{ADVANCED FIRE DETECTION AND INFORMATION SYSTEM}

The Advanced Fire Detection and Information System (AFIS II) was done on two fronts. The first was to upgrade the receiving stations and the second was to improve the software.

New satellite ground receiving stations were installed at the Meraka Institute on the CSIR campus in Pretoria (South Africa) while the original stations at Satellite Application Centre (SAC) at Hartebeeshoek Tracking station would serve as a back-up. The stations at the CSIR in particular would ensure an improvement in the reliability of service [3].

The re-write of the AFIS II system software has been designed to use open standards from the Open Geospatial Consortium (OGC compliant Sensor Web application). This ensures free access to the program code and the ability and flexibility to make changes as they become necessary.

The new AFIS system also combines a number of available database services, such as Google maps as a backdrop. This provides valuable information to the viewer such as background cartography, terrain and 
natural features which will greatly assist in the prediction of the spread of fire.

Interdisciplinary Disaster Risk Reduction Research Centre (IDRRRC), Faculty of Technical Sciences (FTN), University of Novi Sad (UNS) started to work with the South African partner, CSIR MERAKA, to transfer knowledge and experience in the field of fire alert and monitoring, and develop and implement similar system for the "Fruška Gora" National Park's management area, near city of Novi Sad, Vojvodina, Serbia.

When fire is spotted, and pixel generated, an automated e-mail and SMS are sent to the recipient (Fig. 4). E-mail contains the following information:

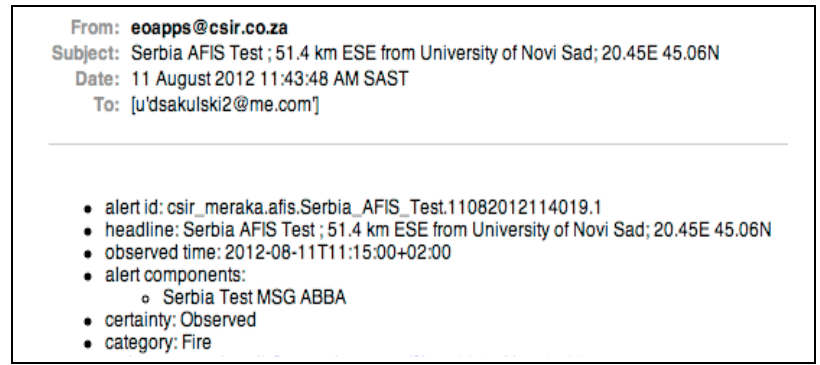

Fig. 4. Automatically generated e-mail informing recipient about fire

\section{- $\quad$ Alert ID}

- Headline pointing to the position of fire (in this example fire was spotted $51.4 \mathrm{~km}$ East-South-East from the University of Novi Sad) including the coordinates
- Date and time (SAST format)

- Certainty

- Category

Fig. 5 shows map of the area around the "Fruška Gora" National Park. Base map is Google map, overlaid by additional layers.

The menu is on the right hand side, containing present day's MODIS and MSG spotted fires, classified into four categories:

- Low intensity

- Medium intensity

- High intensity

- Extreme intensity

Upper button menu enables additional historical data query plus some options in development, such as air quality related information.

\section{CONTINUOUS DEVELOPMENT}

Advanced Fire Information System for the "Fruška Gora" National Park is in process of continuous of development. Workflow of this project consists of the following steps:

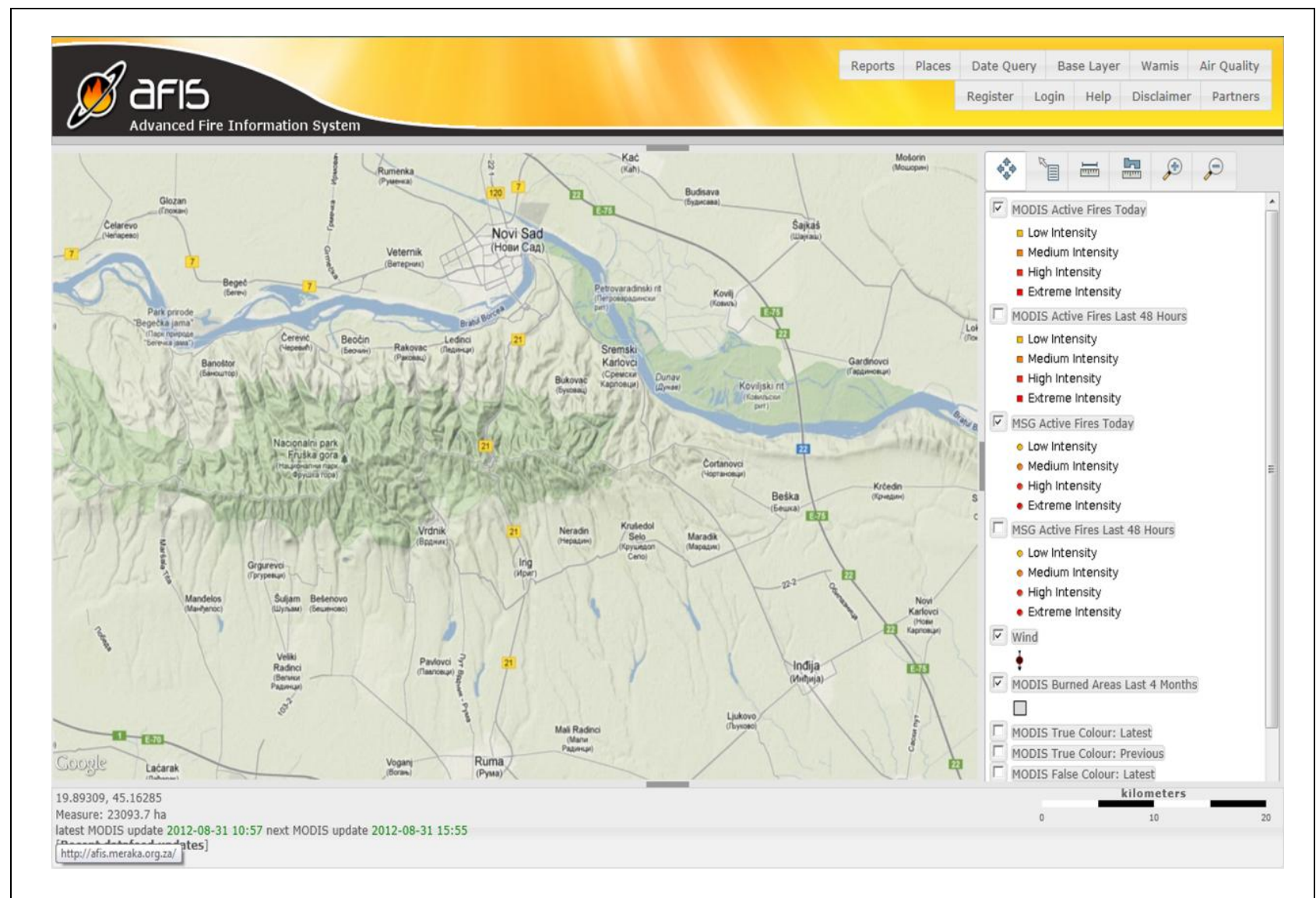

Fig. 5. Screen capture of the pilot AFIS application for the "Fruška Gora" National Park, Vojvodina, Serbia 
- Collecting data, information and experience of a past fires in the region: It is of crucial importance to systematically record every disastrous event.It contributes to the collective memory end enables various spatial and temporal analysis, as well as lessons learned.

- Analyse existing fire monitoring and decision making process: A comprehensive analysis of the existing decision making process regarding fire risk management unveils possible bottlenecks and congestion points. This task should highlight those points and propose solution to either eliminate them or to minimise their negative impact on decision making.

- Analyse structure and information flow of the existing fire information system: Every disaster risk process has the same optimisation goal - minimum time, maximum efficiency. To achieve that goal require comprehensive analysis of flow of data and information. Only basic input data should be in the database. Data relations should be optimised.

- Existing spatial and temporal data availability: Faculty of Technical Sciences'sexpert are already in the process of assessing, collecting and storing relevant available spatial and temporal data regarding National Park management area. Next step will be to organise collection of the remaining relevant spatial data.

- User needs analysis: Questionnaire and interviews will be conducted with the relevant people, as part of the decision making process, to collect optimal amount of information. Those information will be utilise for user needs analysis.

- Information flow analysis: Once the decision making process becomes a clear picture, a flow of information will be analysed to derive an optimal path. It should also highlight possible bottlenecks of such process.

- API modification and improvement: Based on the optimal information flow an application interface will be developed, implemented and tested.

\section{FUTURE RESEARCH PLANS}

Main purpose of this research is to improve leading time of fire detection, location, communication and warning. Presently two satellites are in use (Terra/Aqua Modis) and MeteoSat Second generation (MSG). In the near future additional sensors from the European Constellation of satellites will be also utilized to decrease lead time between fire ignition and satellite detection.

In parallel, mathematical algorithms are being continuously improved to increase fire spatial location and relative information dissemination. An open source software are utilized to visualize additional spatial information such as fire load distribution, location of urban settlements, water bodies, and road network.

\section{REFERENCES}

[1] http://www.eumetsat.int, (2009). European Organization for the Exploitation of Meteorological Satellites (EUMETSAT), Accessed on: 2009-04-06

[2] Davies, D. K.; Vosloo, H. F.; Vannan, S. S. \& Frost, P. E. (2008). Near real-time fire alert system in South Africa: from desktop to mobile service, Proceedings of the 7th ACM conference on designing interactive systems, 25-27th February, Cape Town, South Africa, ISBN 978-1-60558-002-9, Marsden, G.; Ladeira, I. \& Koize P. (Ed.), pp. 315-322, Published by ACM New York, New York

[3] McFerren, G. \& Frost, P. (2009). The Southern African Advanced Fire Information System, Proceedingsof the 6th International ISCRAM Conference, 10-13th May, Gothenburg, Sweden, ISBN 978-91-633-4715-3, Landgren, J. \& Jul, S. (Ed.), pp. 16,Published by University of Gothenburg, Sweden

[4] Lineweber, D. \& McNulty, S. (2001). The cost of Power Disturbances to Industrial and Digital Economy companies, EPRI

[5] Sukhnandan, A. \& Hoch, D.A. (2002): Fire induced Flashovers of Transmission lines: Theoretical models, Proceedings of the 6th AFRICON Conference in Africa, 2-4th October, George, South Africa, ISBN 0-7803-7570, pp. 617-622,.Published by AFRICON, George

[6] Vajeth, R.; Mtolo, D. \& Dama, D. (2003). Cost of a Network Fault Affectings Transmission Supply Point, Eskom Enterprise, RES/RR/03/20237

[7] Vosloo H. F.; Frost, P.; Momberg, A. \& Josephine, I. (2009): Real-time monitoring of the Transmission system: watching out for fires, Proceedings of the 6th Southern Africa Regional Conference, 17-21st August, Somerset West, South Africa 